\title{
Algebraic structures within subsets of Hamel and Sierpiński-Zygmund functions
}

\author{
Krzysztof Płotka
}

\begin{abstract}
We prove the existence of an additive semigroup of cardinality $2^{\mathfrak{c}}$ contained in the intersection of the classes of Hamel functions (HF) and Sierpinski-Zygmund functions (SZ). In addition, we show that under certain set-theoretic assumptions the lineability of the class of Sierpinski-Zygmund functions (SZ) is equal to the lineability of the class of almost continuous Sierpiński-Zygmund functions (AC $\cap \mathrm{SZ}$ ).
\end{abstract}

\section{Introduction}

The symbols $\mathbb{N}, \mathbb{Q}$, and $\mathbb{R}$ denote the sets of positive integers, rational and real numbers, respectively. The cardinality of a set $X$ is denoted by the symbol $|X|$. In particular, $|\mathbb{N}|$ is denoted by $\omega$ and $|\mathbb{R}|$ is denoted by $\mathfrak{c}$. We consider only realvalued functions. No distinction is made between a function and its graph. For any two partial real functions $f, g$ we write $f+g, f-g$ for the sum and difference functions defined on $\operatorname{dom}(f) \cap \operatorname{dom}(g)$. We write $f \mid A$ for the restriction of $f$ to the set $A \subseteq \mathbb{R}$. For any subset $Y$ of a vector space $V$ over the field $E$, any $v \in V$, and any $e \in E$ we define $v+Y=\{v+y: y \in Y\}$ and $e Y=\{e y: y \in Y\}$.

Recently, there have been lots of attention devoted to finding "large" structures (e.g., vector spaces, algebras) contained in various families of real functions (see $[1,3-6,8-10,12,16,18])$. In this article we also consider "less restrictive" structures like groups and even semigroups. In case of many classes of functions the problem is trivially solved by using already known results about vector

Received by the editors in December 2014.

Communicated by F. Bastin.

2010 Mathematics Subject Classification : Primary 15A03; Secondary 26A21, 03 E75.

Key words and phrases : lineability, Hamel functions, Sierpiński-Zygmund functions. 
spaces contained in those classes (as these vector spaces have maximal possible dimensions). However, in certain situations looking for the "largest" group or semigroup may be of interest.

We will recall here some of the most recent definitions related to the theory of lineability (see $[3,5,6])$. Let $V$ be a vector space over the field $E, \mathcal{F} \subseteq V$, and $\kappa$ be a cardinal number. We say that $\mathcal{F}$ is star-like (with respect to $E$ ) if $e \mathcal{F} \subseteq \mathcal{F}$ for all $e \in E \backslash\{0\}$. In addition, $\mathcal{F}$ is defined to be $\kappa$-lineable (over $E$ ) if $\mathcal{F} \cup\{0\}$ contains a subspace of $V$ of dimension $\kappa$. The (coefficient of lineability of the subset $\mathcal{F}$ over the field $E$ is denoted by $\mathcal{L}_{E}(\mathcal{F})$ and defined as follows

$$
\mathcal{L}_{E}(\mathcal{F})=\min \{\kappa: \mathcal{F} \text { is not } \kappa \text {-lineable over } E\} .
$$

In the case $E=\mathbb{R}$ we simply write $\mathcal{L}(\mathcal{F})$.

Proposition 1.1. Let $V$ be a vector space over the field $E_{2}$ and $E_{1}$ be a subfield of $E_{2}$. If $\mathcal{F} \subseteq V$ is star-like with respect to $E_{2}$, then the following holds.

(1)

$$
\mathcal{L}_{E_{1}}(\mathcal{F}) \geq \begin{cases}\left(\left(\mathcal{L}_{E_{2}}(\mathcal{F})-1\right) \cdot \operatorname{dim}_{E_{1}}\left(E_{2}\right)\right)^{+} & , \text {if } \mathcal{L}_{E_{2}}(\mathcal{F})<\omega \\ \mathcal{L}_{E_{2}}(\mathcal{F}) \cdot\left(\operatorname{dim}_{E_{1}}\left(E_{2}\right)\right)^{+} & , \text {otherwise }\end{cases}
$$

(2) If $E_{1}$ is the smallest subfield of $E_{2}$ and $\mathcal{G}$ is an additive group contained in $\mathcal{F} \cup\{0\}$, then $E_{1} \mathcal{G}=\bigcup_{e \in E_{1}} e \mathcal{G}$ is a vector subspace of $V$ over $E_{1}$ contained in $\mathcal{F} \cup\{0\}$.

Proof. (1) Choose any $\kappa<\mathcal{L}_{E_{2}}(\mathcal{F})$ and let $W \subseteq V$ be a subspace over $E_{2}$ contained in $\mathcal{F} \cup\{0\}$ such that $\operatorname{dim}_{E_{2}}(W)=\kappa$. Obviously, $W$ is also a subspace when considered over $E_{1}$ and it can be verified that

$$
\operatorname{dim}_{E_{1}}(W)=\kappa \cdot \operatorname{dim}_{E_{1}}\left(E_{2}\right)
$$

Indeed, if $\left\{f_{\xi}: \xi<\kappa\right\}$ is a basis of $W$ over $E_{2}$, then $\left\{q_{\lambda} f_{\xi}: \xi<\kappa, \lambda<\operatorname{dim}_{E_{1}}\left(E_{2}\right)\right\}$ is a basis of $W$ over $E_{1}$, where $\left\{q_{\lambda}: \lambda<\operatorname{dim}_{E_{1}}\left(E_{2}\right)\right\}$ is a basis of $E_{2}$ over $E_{1}$.

Now, if $\mathcal{L}_{E_{2}}(\mathcal{F})<\omega$ then the largest possible $\kappa$ is $\mathcal{L}_{E_{2}}(\mathcal{F})-1$ and in this case $\operatorname{dim}_{E_{1}}(W)=\left(\mathcal{L}_{E_{2}}(\mathcal{F})-1\right) \cdot \operatorname{dim}_{E_{1}}\left(E_{2}\right)$ and consequently $\mathcal{L}_{E_{1}}(\mathcal{F}) \geq$ $\left(\left(\mathcal{L}_{E_{2}}(\mathcal{F})-1\right) \cdot \operatorname{dim}_{E_{1}}\left(E_{2}\right)\right)^{+}$.

If $\mathcal{L}_{E_{2}}(\mathcal{F}) \geq \omega$, then $\mathcal{L}_{E_{1}}(\mathcal{F}) \geq \max \left\{\mathcal{L}_{E_{2}}(\mathcal{F}),\left(\operatorname{dim}_{E_{1}}\left(E_{2}\right)\right)^{+}\right\}=\mathcal{L}_{E_{2}}(\mathcal{F})$. $\left(\operatorname{dim}_{E_{1}}\left(E_{2}\right)\right)^{+}$.

(2) Since $\mathcal{G} \subseteq \mathcal{F} \cup\{0\}$ and $\mathcal{F}$ is star-like obviously $E_{1} \mathcal{G} \subseteq \mathcal{F} \cup\{0\}$. Additionally, observe that

$$
E_{1}=\left\{ \pm \frac{e_{n}}{e_{k}}: k, n \in \mathbb{Z}_{+} \text {and } e_{k} \neq 0, \text { where } e_{i} \text { is the sum of } i 1^{\prime} \text { s }\right\} \cup\{0\} .
$$

Therefore, for all $g_{1}, g_{2} \in \mathcal{G}$ and $q_{1}, q_{2} \in E_{1} \backslash\{0\}$ we have

$$
q_{1} g_{1}+q_{2} g_{2}= \pm \frac{e_{n_{1}}}{e_{k_{1}}} g_{1} \pm \frac{e_{n_{2}}}{e_{k_{2}}} g_{2}=\frac{1}{e_{k_{1}} e_{k_{2}}}\left( \pm e_{n_{1}} e_{k_{2}} g_{1} \pm e_{n_{2}} e_{k_{1}} g_{2}\right) \in E_{1} \mathcal{G}
$$

Hence, $E_{1} \mathcal{G}$ is a vector subspace of $V$ over $E_{1}$ contained in $\mathcal{F} \cup\{0\}$. 
Observe that in general, the weak inequality in part (1) cannot be replaced by equality neither strict inequality. Indeed, if $\mathcal{F}$ is a vector space over $E_{2}$, then there is equality in part (1). On the other hand if we pick $V=\mathbb{R}^{\mathbb{R}}, E_{1}=\mathbb{Q}, E_{2}=\mathbb{R}$, $B$ to be a basis of $V$ over $E_{2}$, and define $\mathcal{F}=\operatorname{span}_{\mathrm{Q}}(B) \cup \bigcup_{e \in \mathbb{R}} e B$ then we have $\mathcal{L}_{E_{1}}(\mathcal{F})=\left(2^{\mathfrak{c}}\right)^{+}$and $\mathcal{L}_{E_{2}}(\mathcal{F})=2$. Hence in that case there is strict inequality $>$ in part (1).

As a consequence of the above proposition, let us note here that if $E_{1}=\mathbb{Q}$ and $E_{2}=\mathbb{R}$ then for any star-like $\mathcal{F}$ we have that $\mathcal{L}_{\mathbb{Q}}(\mathcal{F}) \geq\left(\operatorname{dim}_{\mathbb{Q}}(\mathbb{R})\right)^{+}=\mathfrak{c}^{+}$. Additionally, every additive group contained in $\mathcal{F} \cup\{0\}$ has cardinality less than $\mathcal{L}_{\mathrm{Q}}(\mathcal{F})$.

In this article we consider the following classes of functions. A function $f: \mathbb{R} \rightarrow \mathbb{R}$ is:

- an extendability function provided there exists a connectivity function $F: \mathbb{R} \times[0,1] \rightarrow \mathbb{R}$ such that $f(x)=F(x, 0)$ for every $x \in \mathbb{R}(f \in$ Ext $) ;$

- almost continuous (in sense of Stallings) if each open subset of $\mathbb{R}^{2}$ containing the graph of $f$ contains also the graph of a continuous function from $\mathbb{R}$ to $\mathbb{R}$ $(f \in \mathrm{AC})$;

- Hamel function if the graph of $f$ is a Hamel basis for $\mathbb{R}^{2}(f \in \mathrm{HF})$;

- Sierpiński-Zygmund if for every set $Y \subseteq \mathbb{R}$ of cardinality continuum $\mathfrak{c}, f \mid Y$ is discontinuous $(f \in \mathrm{SZ})$.

Recall here that the class of all continuous functions is contained in Ext, Ext $\subseteq$ $\mathrm{AC}, \mathrm{Ext} \cap \mathrm{SZ}=\varnothing, \mathrm{AC} \cap \mathrm{SZ} \neq \varnothing$ under additional set-theoretical assumptions (e.g., $\mathrm{CH}$, Martin's Axiom), Ext $\cap \mathrm{HF} \neq \varnothing, \mathrm{AC} \cap \mathrm{HF} \neq \varnothing$, and $\mathrm{HF} \cap \mathrm{SZ} \neq \varnothing$ (see [17]). In addition, a function $f: \mathbb{R} \rightarrow \mathbb{R}$ is almost continuous if and only if it intersects every blocking set, i.e., a closed set $K \subseteq \mathbb{R}^{2}$ which meets every continuous function and is disjoint with at least one function from $\mathbb{R}$ to $\mathbb{R}$. The domain of every blocking set contains a non-degenerate connected set. (See [11].) For $f \in \mathcal{F} \subseteq \mathbb{R}^{\mathbb{R}}$ we say that a set $A \subseteq \mathbb{R}$ is $f$-negligible with respect to $\mathcal{F}$ if for every function $g$ such that $f|(\mathbb{R} \backslash A) \equiv g|(\mathbb{R} \backslash A)$ we have that $g \in \mathcal{F}$.

It is known that $\mathcal{L}(\mathrm{SZ})>\mathfrak{c}^{+}$(see [9]) and that $2^{\mathfrak{c}}$-lineability of SZ is undecidable in ZFC (see [10]). In [10] the authors also proved (Theorem 2.2) that for any $\mathfrak{c}<\kappa \leq 2^{\mathfrak{c}}, \mathcal{L}(\mathrm{SZ})>\kappa$ is equivalent to the existence of an additive group in $S Z \cup\{0\}$ of cardinality $\kappa$. This immediately implies the following property.

Remark 1.2. $\mathcal{L}_{\mathrm{Q}}(\mathrm{SZ})=\mathcal{L}(\mathrm{SZ})$.

In the case of Hamel functions we have the following: $\mathcal{L}(\mathrm{HF})=2$ and $\mathcal{L}_{\mathbb{Q}}(\mathrm{HF})=\mathfrak{c}^{+}($see $[18])$. 


\section{Semigroup in $\mathrm{HF} \cap S Z$ and lineability of $A C \cap S Z$}

We will be using the following two lemmas to prove the existence of "large" semigroups in $\mathrm{HF} \cap \mathrm{SZ}$, Ext $\cap \mathrm{HF}$, and $\mathrm{AC} \cap \mathrm{HF} \cap \mathrm{SZ}$ (under the assumption of $\mathrm{CH}$ ).

Lemma 2.1. [17, Lemma 7] Let $V \subseteq \mathbb{R}^{n}$ be a Hamel basis and $v^{\prime} \in V$. For each $v \in V$ fix $q_{v} \in \mathbb{Q}$ such that $q_{v^{\prime}} \neq-1$. Then the set $V^{\prime}=\left\{v+q_{v} v^{\prime}: v \in V\right\}$ is also a Hamel basis.

Lemma 2.2. There exists a function $h \in H F \cap$ Ext and a set $X \subseteq \mathbb{R}$ of cardinality $\mathfrak{c}$ which is h-negligible with respect to Ext. Assuming $\mathrm{CH}$, there exists a function $h \in \mathrm{AC} \cap \mathrm{HF} \cap \mathrm{SZ}$ and a set $X \subseteq \mathbb{R}$ of cardinality $\mathfrak{c}$ which is $h$-negligible with respect to $\mathrm{AC}$.

Proof. Let $F \subseteq \mathbb{R}$ be a linearly independent $\mathfrak{c}$-dense $F_{\sigma}$ set (see [14, Theorem 11.7.2]). Then there exists a function $f \in$ Ext such that $\mathbb{R} \backslash F$ is $f$-negligible (see [7]). Using [17, Fact 6] we obtain the existence of a function $h \in$ HF such that $h|F \equiv f| F$. Obviously, $h \in$ Ext and $X=\mathbb{R} \backslash F$ is $h$-negligible with respect to Ext.

In the proof of Theorem 2 in [17] (page 123) a function $h$ is constructed which belongs to $\mathrm{AC} \cap \mathrm{HF} \cap \mathrm{SZ}$ (under $\mathrm{CH}$ ). One can easily see that this function $h$ has a dense graph. It is known that for an almost continuous function $f$ with a dense graph, every nowhere dense set is $f$-negligible with respect to AC (see [13]).

Theorem 2.3. Both $\mathrm{HF} \cap \mathrm{Ext}$ and $\mathrm{HF} \cap \mathrm{SZ}$ contain an additive semigroup of size $2^{\mathfrak{c}}$. In addition, assuming $\mathrm{CH}$, the same holds for $\mathrm{AC} \cap \mathrm{HF} \cap \mathrm{SZ}$.

Proof. We will prove the statement for the family AC $\cap \mathrm{HF} \cap \mathrm{SZ}$. By the previous lemma, under the assumption of $\mathrm{CH}$ there exists a function $h \in \mathrm{AC} \cap \mathrm{HF} \cap \mathrm{SZ}$ and a set $X \subseteq \mathbb{R}$ of cardinality $\mathfrak{c}$ which is $h$-negligible with respect to AC. Define $\mathcal{H}=\left\{q h+h(0) g: q \in \mathbb{Q}_{+}, g \in \mathbb{Q}_{+}(X)\right\}$ where $\mathbb{Q}_{+}$is the set of positive rationals and $\mathbb{Q}_{+}(X)=\left\{f \in \mathbb{R}^{\mathbb{R}}: f \mid(\mathbb{R} \backslash X) \equiv 0\right.$ and $f(x) \in \mathbb{Q}_{+}$for $\left.x \in X\right\}$. Since $h(0) \neq 0$ (for every $f \in \mathrm{HF}, f(0) \neq 0$ ) we conclude that $|\mathcal{H}|=2^{\mathfrak{c}}$. Next observe that $\mathcal{H}$ is closed under addition as both $\mathbb{Q}_{+}$and $\mathbb{Q}_{+}(X)$ are closed under addition.

Finally we will justify that $\mathcal{H} \subseteq \mathrm{AC} \cap \mathrm{HF} \cap \mathrm{SZ}$. Obviously $\mathcal{H} \subseteq \mathrm{AC}$ as $\mathrm{AC}$ is star-like and $X$ is $h$-negligible with respect to $\mathrm{AC}$. To see $\mathcal{H} \subseteq \mathrm{HF}$ recall that $\mathrm{HF}$ is star-like and then use Lemma 2.1 with $V=h, v^{\prime}=(0, h(0))$, and $q_{v}=\frac{g(x)}{q}$ for $v=(x, h(x)), q \in \mathbb{Q}_{+}, g \in \mathbb{Q}_{+}(X)$ to conclude that $h+h(0) \frac{g}{q}$ is a Hamel function $\left(h+h(0) \frac{g}{q}\right.$ is $V^{\prime}$ from Lemma 2.1). Consequently, $q h+h(0) g=q\left(h+h(0) \frac{g}{q}\right) \in$ HF.

To see $\mathcal{H} \subseteq \mathrm{SZ}$ recall that SZ is star-like and observe that $h(0) g$ is a countably continuous function (e.g., union of countably many partial continuous functions) for all $g \in \mathbb{Q}_{+}(X)$. This implies $q h+h(0) g \in \mathrm{SZ}$.

The existence of semigroups of cardinality $2^{\mathfrak{c}}$ in $\mathrm{HF} \cap \mathrm{Ext}$ and $\mathrm{HF} \cap \mathrm{SZ}$ can be justified in a very similar way (in the case of $H F \cap S Z$ use $X=\mathbb{R}$ ). 
Theorem 2.4. Assume $\mathrm{CH}$. Then $\mathcal{L}(\mathrm{AC} \cap \mathrm{SZ})>\mathfrak{c}^{+}$.

Proof. Let $\mathcal{F}=\left\{f_{\gamma}: \gamma<\mathfrak{c}\right\} \subseteq(\mathrm{AC} \cap \mathrm{SZ}) \cup\{0\}$ be a vector space of dimension $\leq$ c. We will show that there exists an $h \in \mathrm{AC} \cap \mathrm{SZ} \backslash \mathcal{F}$ such that $h+\mathcal{F} \subseteq \mathrm{AC} \cap \mathrm{SZ}$. Since AC $\cap \mathrm{SZ}$ is star-like the latter will imply that $\{a h: a \in \mathbb{R}\}+\mathcal{F}$ is a vector space in $(\mathrm{AC} \cap \mathrm{SZ}) \cup\{0\}$ such that $\mathcal{F} \subsetneq\{a h: a \in \mathbb{R}\}+\mathcal{F}$. Next using Zorn's lemma we will be able to conclude that $(\mathrm{AC} \cap \mathrm{SZ}) \cup\{0\}$ contains a vector space of dimension $\mathfrak{c}^{+}$.

Let $\mathcal{G}=\left\{g_{\alpha}: \alpha<\mathfrak{c}\right\}$ be the set of all continuous functions defined on $G_{\delta}$ subsets of $\mathbb{R}=\left\{x_{\alpha}: \alpha<\mathfrak{c}\right\}$. For every $\alpha<\mathfrak{c}$ define $U_{\alpha}$ to be the maximal open set such that $\operatorname{dom}\left(g_{\alpha} \backslash \bigcup_{\xi<\alpha} g_{\xi}\right)$ is residual in $U_{\alpha}$. We will construct by induction a sequence of partial functions $h_{\alpha}(\alpha<\mathfrak{c})$ such that:

(i) $h_{\xi} \subseteq h_{\alpha}$ for $\xi<\alpha$;

(ii) $\left|\operatorname{dom}\left(h_{\alpha}\right)\right| \leq \omega$ and $x_{\alpha} \in \operatorname{dom}\left(h_{\alpha}\right)$;

(iii) $\left(g_{\zeta} \cap\left(f_{\gamma}+h_{\alpha}\right)\right) \subseteq\left(f_{\gamma}+h_{\xi}\right)$ for $\zeta, \gamma \leq \xi<\alpha$;

(iv) $f_{\gamma}+h_{\alpha}$ is dense subset of $\left(g_{\zeta} \backslash \bigcup_{\xi<\zeta} g_{\xi}\right) \mid U_{\zeta}$ for $\zeta, \gamma \leq \alpha$.

We start the construction of the sequence $h_{\alpha}(\alpha<\mathfrak{c})$ by defining $h_{0}\left(x_{0}\right)$ arbitrarily. Next choose a countable dense subset $D_{0} \subseteq\left(\operatorname{dom}\left(g_{0}\right) \cap U_{0}\right) \backslash\left\{x_{0}\right\}$ and put $\left(f_{0}+h_{0}\right)\left|D_{0} \equiv g_{0}\right| D_{0}$ (or equivalently $\left.h_{0}\left|D_{0} \equiv\left(g_{0}-f_{0}\right)\right| D_{0}\right)$. It is easy to see that $h_{0}$ satisfies all the conditions (i)-(iv).

Now fix $\alpha<\mathfrak{c}$ and assume that the sequence $h_{\beta}$ has been defined for all $\beta<\alpha$ satisfying the conditions (i)-(iv). Put $h_{\alpha}=\bigcup_{\beta<\alpha} h_{\beta}$. If $x_{\alpha} \notin \operatorname{dom}\left(h_{\alpha}\right)$, then choose

$$
\left.h_{\alpha}\left(x_{\alpha}\right) \in \mathbb{R} \backslash \bigcup_{\gamma, \beta<\alpha}\left\{g_{\beta}\left(x_{\alpha}\right)-f_{\gamma}\left(x_{\alpha}\right)\right\}\right) .
$$

Next notice that since the conditions (i)-(iv) are satisfied for all $\beta<\alpha$ to have that

$$
f_{\gamma}+h_{\alpha} \text { is dense in }\left(g_{\zeta} \backslash \bigcup_{\zeta<\zeta} g_{\xi}\right) \mid U_{\zeta} \text { for } \zeta, \gamma \leq \alpha
$$

it suffices to assure the above condition for $\zeta=\alpha$ or $\gamma=\alpha$. Choose a collection of pairwise disjoint countable sets $D_{\gamma, \xi}((\gamma<\alpha$ and $\xi=\alpha)$ or $(\gamma=\alpha$ and $\xi \leq \alpha))$ contained in

$$
\mathbb{R} \backslash\left(\operatorname{dom}\left(h_{\alpha}\right) \cup \bigcup_{\tilde{\xi}_{1}, \tilde{\xi}_{2}, \gamma_{1}, \gamma_{2} \leq \alpha, \gamma_{1} \neq \gamma_{2}} \operatorname{dom}\left(\left(g_{\xi_{1}}-g_{\xi_{2}}\right) \cap\left(f_{\gamma_{1}}-f_{\gamma_{2}}\right)\right)\right),
$$

such that $D_{\gamma, \alpha}$ is dense subset of $\operatorname{dom}\left(g_{\alpha} \backslash \bigcup_{\beta<\alpha} g_{\beta}\right) \cap U_{\alpha}(\gamma<\alpha)$ and $D_{\alpha, \xi}$ is dense subset of $\operatorname{dom}\left(g_{\xi} \backslash \bigcup_{\beta<\xi} g_{\beta}\right) \cap U_{\xi}(\xi \leq \alpha)$. Note here that the above choice is possible as $\left|\operatorname{dom}\left(\left(g_{\xi_{1}}-g_{\xi_{2}}\right) \cap\left(f_{\gamma_{1}}-f_{\gamma_{2}}\right)\right)\right| \leq \omega$ for $\xi_{1}, \xi_{2}, \gamma_{1}, \gamma_{2} \leq \alpha, \gamma_{1} \neq \gamma_{2}$ because $g_{\xi_{1}}-g_{\xi_{2}}$ is a continuous function, $f_{\gamma_{1}}-f_{\gamma_{2}} \in \mathrm{SZ}$, and we work under the assumption of $\mathrm{CH}$. Now we define $f_{\gamma}+h_{\alpha}\left|D_{\gamma, \alpha} \equiv g_{\alpha}\right| D_{\gamma, \alpha}$ for $\gamma<\alpha$ and 
$f_{\alpha}+h_{\alpha}\left|D_{\alpha, \xi} \equiv g_{\xi}\right| D_{\alpha, \xi}$ for $\xi \leq \alpha$. This finishes the construction of $h_{\alpha}$. It is clear that $h_{\alpha}$ satisfies the conditions (i), (ii), and (iv).

To see that the condition (iii) is also satisfied let us pick $\xi<\alpha$ and $\zeta, \gamma \leq \xi$. By the inductive assumption we obtain that $\left(g_{\zeta} \cap\left(f_{\gamma}+\bigcup_{\beta<\alpha} h_{\beta}\right)\right) \subseteq$ $\left(f_{\gamma}+h_{\xi}\right)$. Therefore to conclude that $\left(g_{\zeta} \cap\left(f_{\gamma}+h_{\alpha}\right)\right) \subseteq\left(f_{\gamma}+h_{\xi}\right)$ we need to justify that $g_{\zeta} \cap\left(f_{\gamma}+h_{\alpha} \mid\left\{x_{\alpha}\right\}\right)=\varnothing, g_{\zeta} \cap\left(f_{\gamma}+h_{\alpha} \mid D_{\gamma_{1}, \alpha}\right)=\varnothing\left(\gamma_{1}<\alpha\right)$, and $g_{\zeta} \cap\left(f_{\gamma}+h_{\alpha} \mid D_{\alpha, \xi}\right)=\varnothing(\xi \leq \alpha)$. The equality $g_{\zeta} \cap\left(f_{\gamma}+h_{\alpha} \mid\left\{x_{\alpha}\right\}\right)=\varnothing$ easily follows from the definition of $h_{\alpha}\left(x_{\alpha}\right)$. To see $g_{\zeta} \cap\left(f_{\gamma}+h_{\alpha} \mid D_{\gamma_{1}, \alpha}\right)=\varnothing\left(\gamma_{1}<\alpha\right)$ note that $g_{\zeta} \cap\left(f_{\gamma}+h_{\alpha} \mid D_{\gamma_{1}, \alpha}\right)=g_{\zeta} \cap\left(f_{\gamma}-f_{\gamma_{1}}+g_{\alpha}\right) \mid D_{\gamma_{1}, \alpha}$. If $\gamma=\gamma_{1}$, then $g_{\zeta} \cap\left(f_{\gamma}+h_{\alpha} \mid D_{\gamma_{1}, \alpha}\right)=g_{\zeta} \cap g_{\alpha} \mid D_{\gamma_{1}, \alpha}=\varnothing$ as $D_{\gamma_{1}, \alpha} \subseteq \operatorname{dom}\left(g_{\alpha} \backslash \bigcup_{\beta<\alpha} g_{\beta}\right)$. If $\gamma \neq \gamma_{1}$, then $g_{\zeta} \cap\left(f_{\gamma}+h_{\alpha} \mid D_{\gamma_{1}, \alpha}\right)=\left(g_{\zeta}-g_{\alpha}\right) \cap\left(f_{\gamma}-f_{\gamma_{1}}\right) \mid D_{\gamma_{1}, \alpha}=\varnothing$ as

$$
D_{\gamma_{1}, \alpha} \cap \bigcup_{\xi_{1}, \xi_{2}, \gamma_{1}, \gamma_{2} \leq \alpha, \gamma_{1} \neq \gamma_{2}} \operatorname{dom}\left(\left(g_{\xi_{1}}-g_{\xi_{2}}\right) \cap\left(f_{\gamma_{1}}-f_{\gamma_{2}}\right)\right)=\varnothing .
$$

Very similarly we can justify that $g_{\zeta} \cap\left(f_{\gamma}+h_{\alpha} \mid D_{\alpha, \xi}\right)=\varnothing(\xi \leq \alpha)$. Hence the condition (iii) holds for $h_{\alpha}$. This finishes the inductive definition of the sequence $h_{\alpha}(\alpha<\mathfrak{c})$ satisfying the conditions (i)-(iv).

Define $h=\bigcup_{\alpha<\mathfrak{c}} h_{\alpha}$. Obviously $\operatorname{dom}(h)=\mathbb{R}$. The conditions (ii)-(iii) imply that $h+f_{\gamma} \in \mathrm{SZ}$ for all $\gamma<\mathfrak{c}$ as any partial continuous function can be extended to a continuous function on a $G_{\delta}$ subset of $\mathbb{R}$ (see [15]) and $\left(g_{\zeta} \cap\left(f_{\gamma}+h\right)\right) \subseteq$ $\left(f_{\gamma}+h_{\max (\zeta, \gamma)}\right)$ for all $\zeta<\mathfrak{c}$.

Next we will argue that $h+f_{\gamma}$ is almost continuous for every $\gamma<\mathfrak{c}$. Let $B \subseteq \mathbb{R}^{2}$ be any blocking set. There exists a non-empty open interval $I \subseteq \operatorname{dom}(B)$ and a continuous function $g$ such that $\operatorname{dom}(g)$ is $G_{\delta}$ dense subset of $I$ and $g \subseteq B$. Let $\zeta_{0}$ be the smallest ordinal number with the property that $g_{\zeta_{0}} \mid I \subseteq B$ and $\operatorname{dom}\left(g_{\zeta_{0}}\right) \cap I$ is residual in $I$ for some non-empty open interval $I \subseteq \operatorname{dom}(B)$. Then $\operatorname{dom}\left(g_{\zeta_{0}} \backslash \bigcup_{\xi<\zeta_{0}} g_{\xi}\right)$ is also residual in $I$ (since we assume $\mathrm{CH}$ ). Therefore, $I \subseteq U_{\zeta_{0}}$ and since $f_{\gamma}+h$ is dense subset of $\left(g_{\zeta_{0}} \backslash \bigcup_{\xi<\zeta_{0}} g_{\xi}\right) \mid U_{\zeta_{0}}$ (condition (iv) for $\left.\alpha=\max \left(\gamma, \zeta_{0}\right)\right)$ we obtain that

$$
\varnothing \neq\left(h+f_{\gamma}\right) \cap\left(g_{\zeta_{0}} \backslash \bigcup_{\xi<\zeta_{0}} g_{\xi}\right)\left|I \subseteq\left(h+f_{\gamma}\right) \cap g_{\zeta_{0}}\right| I \subseteq\left(h+f_{\gamma}\right) \cap B .
$$

This implies that $h+f_{\gamma} \in \mathrm{AC}$.

Let us mention here that assuming GHC the above theorem implies that $\mathrm{AC} \cap \mathrm{SZ}$ is $2^{\mathfrak{c}}$-lineable and consequently $\mathcal{L}(\mathrm{AC} \cap \mathrm{SZ})=\mathcal{L}(\mathrm{SZ})$. On the other hand, there is a model of ZFC (see [2]) in which $\mathrm{AC} \cap \mathrm{SZ}=\varnothing$. These two observations imply the following.

\section{Corollary 2.5.}

(1) It is consistent with $Z F C$ that $\mathcal{L}(\mathrm{AC} \cap \mathrm{SZ})=\mathcal{L}(\mathrm{SZ})$.

(2) It is consistent with $\mathrm{ZFC}$ that $\mathcal{L}(\mathrm{AC} \cap \mathrm{SZ})<\mathcal{L}(\mathrm{SZ})$.

It would be interesting to know if it is possible to have $A C \cap S Z \neq \varnothing$ and $\mathcal{L}(\mathrm{AC} \cap \mathrm{SZ})<\mathcal{L}(\mathrm{SZ})$. We state that as an open problem.

Problem 2.6. Is it consistent with $\mathrm{ZFC}$ that $\mathrm{AC} \cap \mathrm{SZ} \neq \varnothing$ and $\mathcal{L}(\mathrm{AC} \cap \mathrm{SZ})<$ $\mathcal{L}(\mathrm{SZ})$ ? 


\section{References}

[1] R.M. Aron, V.I. Gurariy, J.B. Seoane-Sepúlveda, Lineability and spaceability of sets of functions on $\mathbb{R}$, Proc. Amer. Math. Soc. 133(3) (2005), 795-803.

[2] M. Balcerzak, K. Ciesielski, T. Natkaniec, Sierpinski-Zygmund functions that are Darboux, almost continuous, or have a perfect road, Arch. Math. Logic 37 (1997), 29-35.

[3] A. Bartoszewicz, S. Głąb, Additivity and lineability in vector spaces, Linear Algebra Appl. 439(7) (2013), 2123-2130.

[4] L. Bernal-González, M. Ordóñez Cabrera,Lineability criteria, with applications, J. Funct. Anal. 266(6) (2014), 3997-4025.

[5] L. Bernal-González, D. Pellegrino, J. B. Seoane-Sepúlveda, Linear subsets of nonlinear sets in topological vector spaces, Bull. Amer. Math. Soc. (N.S.) 51(1) (2014), 71-130.

[6] K.C. Ciesielski, J.L. Gámez-Merino, D. Pellegrino, and J.B. SeoaneSepúlveda, Lineability, spaceability, and additivity cardinals for Darboux-like functions, Linear Algebra Appl. 440 (2014), 307-317

[7] K. Ciesielski, J. Jastrzȩbski, Darboux-like functions within the classes of Baire one, Baire two, and additive functions, Topology Appl. (103) (2000), 203-219.

[8] J.L. Gámez-Merino, Large algebraic structures inside the set of surjective functions, Bull. Belg. Math. Soc. Simon Stevin 18(2) (2011), 297-300.

[9] J.L. Gámez-Merino, G.A. Muñoz-Fernández, V.M. Sanchez, J.B. SeoaneSepúlveda, Sierpiński-Zygmund functions and other problems on lineability, Proc. Amer. Math. Soc. 138 (11) (2010), 3863-3876.

[10] J. L. Gámez-Merino, J. B. Seoane-Sepúlveda, An undecidable case of lineability in $\mathbb{R}^{\mathbb{R}}$, J. Math. Anal. Appl. 401(2) (2013), 959-962.

[11] R.G. Gibson, T. Natkaniec, Darboux-like functions, Real Anal. Exchange 22(2) (1996-97), 492-533.

[12] V.I. Gurariy, L.Quarta, On lineability of sets of continuous functions, J. Math. Anal. Appl. 294 (2004), 62-72.

[13] K.R. Kellum, Almost continuity and connectivity - sometimes it's as easy to prove stronger results, Real Anal. Exchange (8) (1982-1983), 244-252.

[14] M. Kuczma, An Introduction to the Theory of Functional Equations and Inequalities, Birkhäuser Verlag AG, 2009.

[15] K. Kuratowski, Topologie I, Warszawa, 1958.

[16] T. Natkaniec, Algebrability of some families of Darboux-like functions, Linear Algebra Appl. 439 (2013), 3256-3263. 
[17] K. Płotka, Darboux-like functions within the class of Hamel functions, Real Anal. Exchange 34(1) (2009), 115-126.

[18] K. Płotka, On lineability and additivity of functions with finite preimages, J. Math. Anal. Appl. 421(2) (2015), 1396-1404.

Department of Mathematics, University of Scranton, Scranton, PA 18510, USA email:Krzysztof.Plotka@scranton.edu 